Check for updates

Cite this: Chem. Commun., 2017, 53, 4096

Received 23rd February 2017, Accepted 10th March 2017

DOI: $10.1039 / c 7 c c 01434 a$

rsc.li/chemcomm

\section{Nontoxic organic solvents identified using an a priori approach with Hansen solubility parameters $\dagger$}

\author{
Daisuke Nakamura, (D) * Minoru Hirano and Riichiro Ohta (D)
}

\begin{abstract}
Nontoxicities of organic solvents were predicted using Hansen solubility parameters and various truly nontoxic solvents were identified according to the prediction. The nontoxicity of solvents originates from their exhibition of poor solubility or low diffusivity toward cell constituents and good stability against hydrolytic degradation.
\end{abstract}

Materials utilized in medical and biological engineering must be strictly biocompatible. Although some solid materials, such as hydroxyapatite and titanium compounds, exhibit excellent biocompatibility, most pure liquid substances are not biocompatible. Hereafter, the term "biocompatible" or "nontoxic" means "nontoxic to bare cells without any protective layer, such as a mucous membrane or stratum corneum". For example, pure liquid water is highly cytotoxic due to its hypo-osmolality, despite being the primary component of the cell interior. Organic solvents are generally considered as highly cytotoxic liquids ${ }^{1-6}$ and nontoxic organic solvents have not been well investigated, with several exceptions. $^{7-9}$ If nontoxic organic solvents are not exceptional but are simply the result of their physical properties, these solvents would answer a fundamental question: "what is the origin of cytotoxicity?" These solvents would be useful as media for hydrophobic and non-water-dispersible substances/particles for investigating the interactions of these substances/particles with cells. Furthermore, these solvents could be useful as carrier fluids for the 3D printing of living cells ${ }^{10}$ (quick-drying cell ink can be prepared if living cells are dispersed in nontoxic organic solvents) or the fabrication of cell sheets ${ }^{11}$ (cell sheet production efficiency will be improved if cell sheets are cultured at the interface between a liquid growth medium and nontoxic organic solvents).

Here we propose a simple hypothesis explaining nontoxicity/ cytotoxicity in order to efficiently identify nontoxic organic solvents: namely, nontoxic solvents do not dissolve any cell

Toyota Central R\&D Labs., Inc., Nagakute, Aichi 480-1192, Japan.

E-mail:daisuke@mosk.tytlabs.co.jp

$\dagger$ Electronic supplementary information (ESI) available. See DOI: 10.1039/c7cc01434a constituent and/or do not dissolve into cells. If this hypothesis is true, we can a priori predict potentially nontoxic organic solvents via a physical property, namely, Hansen solubility parameters ${ }^{12}$ (HSP) based on the "like dissolves like" concept well established in the field of polymer science.

In this study, we determined the cell-constituent HSP region as the sum set of HSP spheres (see the ESI $\dagger$ for details and limitations of HSP) of individual cell constituents. A complement of this region is presumed to be the nontoxic HSP region, which is the region outside the cell-constituent HSP region. Potentially nontoxic organic solvents (test solvents) are chosen from within and near the presumed nontoxic region. We carried out directcontact tests of the identified pure test solvents to bare cells and found that various solvents are inherently nontoxic, e.g., fluorine compounds such as hydrofluoroethers (HFE), siloxanes, and hydrocarbons (including the major components of diesel fuels and engine oils). Moreover, we carefully examined nontoxic/ cytotoxic solvents in terms of HSPs and other properties and clarified that the nontoxicity of organic solvents originates from being (1) non-compatible (in terms of their HSPs) towards cell constituents and (2) exhibiting high stability towards hydrolysis.

Seven major cell constituents were considered and the cellconstituent identifiers (IDs, C1-C7) were: C1: cholesterol; C2: deoxyribonucleic acids (DNA); C3: phosphatidylcholine; C4: phosphatidylethanolamine; C5: sphingomyelin; C6: phosphatidylserine and C7: water (see Table S1 (ESI $\dagger$ ) for their HSPs). The HSP values of $\mathrm{C} 1, \mathrm{C} 2$ and $\mathrm{C} 7$ are available from the literature, ${ }^{12,13}$ whereas the HSP values of the others (C3-C6: cell-membrane constituents) were estimated using commercial software (HSPiP v4.1.03). ${ }^{14}$ The values for $R_{0}$ listed in Table $\mathrm{S} 1$ are interaction radii and indicate the thresholds for a cell constituent to dissolve in a given solvent (or for a cell constituent to absorb a given solvent) when the HSP distance $R_{\mathrm{a}}$ (see ESI $\dagger$ ) between the constituent and solvent is smaller than $R_{0}{ }^{12}$ Although $R_{0}$ should be experimentally determined, the $R_{0}$ values for C3-C6 were set to $10\left(\mathrm{~J} \mathrm{~cm}^{-3}\right)^{1 / 2}$ by assuming they are similar to the $R_{0}$ values for other cell constituents (cholesterol and DNA, see the ESI $\dagger$ for validity assessment). 

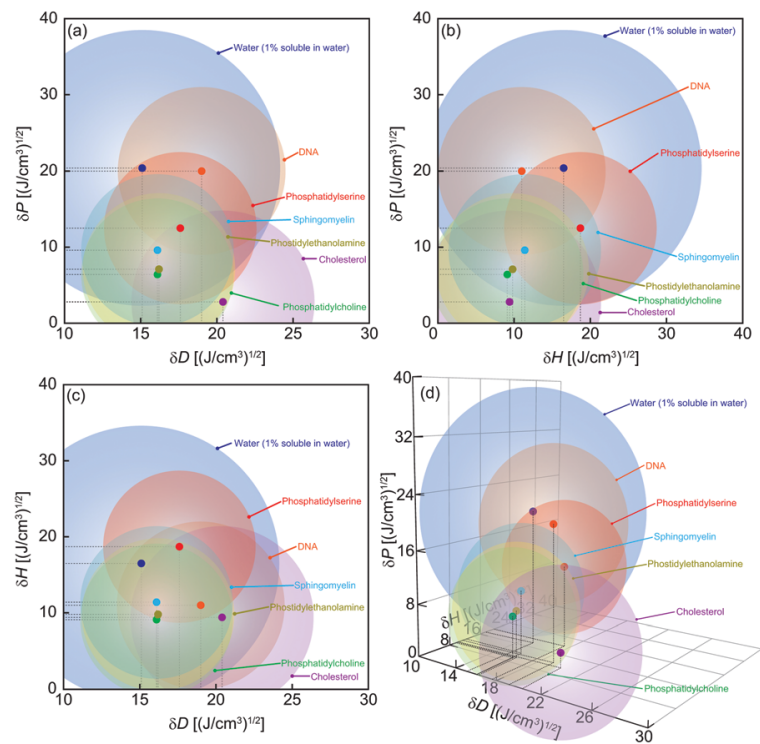

Fig. 1 Plots of HSP spheres of cell constituents in HSP space showing different views: (a) projection views to $\delta D-\delta P$, (b) $\delta H-\delta P$, (c) $\delta D-\delta H$ planes and (d) quasi-3D view.

Fig. 1 shows plots of HSP spheres (centers: HSP values, radii: $R_{0}$ ) of the 7 cell constituents with Cartesian coordinates $\delta D, \delta P$ and $\delta H$ (HSP space). Fig. 1a-c show $2 \mathrm{D}$ views projected onto the $\delta D-\delta P, \delta H-\delta P$ and $\delta D-\delta H$ planes, respectively, and Fig. 1d shows a quasi-3D view. As is evident from Fig. 1, the HSP space is mostly filled with cell-constituents occupying the HSP region, and the complementary region is quite limited. Although a larger HSP region $\left(\delta D>23, \delta P>30\right.$, or $\left.\delta H>30\left(\mathrm{~J} \mathrm{~cm}^{-3}\right)^{1 / 2}\right)$, for example, seems to be a broad empty space, there is no solvent in a liquid state in this region under standard conditions $\left(25^{\circ} \mathrm{C}, 100 \mathrm{kPa}\right)$. Fig. 1a and d suggest the presence of a tight complementary region in a smaller HSP region $(\delta D<16$, $\delta P<8$, and $\left.\delta H<8\left(\mathrm{~J} \mathrm{~cm}^{-3}\right)^{1 / 2}\right)$. By reference to a HSP database of values within and near the complementary region, we chose potentially nontoxic organic solvents that are liquids under standard conditions. The chosen solvents were low-polarity or nonpolar compounds such as fluorine compounds, siloxanes and hydrocarbons.

The cytotoxicities of potentially nontoxic solvents (25 test solvents, IDs S1-S25, Tables S2 and S3, ESI $\dagger$ ) were investigated using a direct-contact test (Fig. S1, ESI $\dagger$ ). In addition, the positive (cytotoxic) controls water, ethanol, acetone and diethylether (IDs: P1-P4), and the three nontoxic solutions RPMI-1640 liquid cell culture medium with $0.5 \mathrm{vol} \%$ fetal bovine serum (FBS), phosphate buffered saline (PBS), and physiological saline (saline) (IDs: N1-N3), were tested for comparison and normalization of the resultant cell survival rates.

The direct-contact tests were carried out by soaking human airway epithelial cells ${ }^{15}$ (BEAS-2B) in test wells with undiluted pure test solvents, typically for $2 \mathrm{~h}$. Afterwards, the test solvents were replaced with RPMI-1640 culture medium containing 0.5 vol\%-FBS, then the cells were incubated for $20 \mathrm{~h}$ to detect delayed cytotoxicity. After this post-test incubation, a dehydrogenase activity test ${ }^{16}$ (WST-8 assay) was conducted to evaluate cell survival rates, and optical-microscope observation of the cell morphologies was carried out. The cell survival rates were normalized to the results obtained using a nontoxic solution. Errors in the cell survival rates were evaluated using the standard errors from the dispersion of 4-well results. For selected test solvents, the WST-8 assay was followed by live/dead cell observation using live/dead staining. ${ }^{17}$

Fig. 2a shows normalized cell survival rates (NCSR) after the direct-contact test and post-test incubation. The results for the test solvents range from highly cytotoxic (NCSR: $\sim 0)$ to nontoxic (NCSR: 1) and all the positive controls (P1-P4) indicate high cytotoxicity (NCSR: 0) as expected. Applying a threshold value of NCSR $>0.8$ for nontoxic solvents (assuming that the overall experimental error was \pm 0.2 , since the maximum NCSR was 1.2), 15 test solvents (S6-S10, S12-S15, S19-S23 and S25) were deemed nontoxic. The nontoxic solvents comprise various chemical species, including fluorine compounds, siloxanes, and hydrocarbons, indicating that nontoxicity is not specific to a particular chemical structure, functional group or atomic element. Furthermore, the probability of identifying nontoxic solvents from potentially nontoxic test solvents (15 out of 25) was rather high. These findings indicate that our hypothesis, namely, that nontoxic organic solvents should occupy the complementary region of the cell-constituent HSP region, is quite probable. In other words, screening on the basis of consideration of the HSPs is useful to effectively identify biocompatible substances. However, S2: perfluorohexane, predicted to be nontoxic by HSP, was in fact moderately cytotoxic (NCSR: 0.28) and may be an anomaly. The existence of anomalies suggests that secondary cytotoxic factors (e.g., a decrease in cell number in the absence of cell death, or cell death unrelated to the test solvent) other than the primary factor of cell-constituent HSPs affected the results. 
Fig. $2 \mathrm{~b}-\mathrm{g}$ show the morphologies of cells after the directcontact test with the solvents/solutions N1, P1, P2, S7 (HFE-

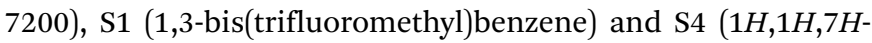
dodecafluoro-1-heptanol) (see other results in Fig. S3-S5, ESI $\dagger$ ). The morphologies of cells tested using a known nontoxic solution and nontoxic test solvent (Fig. $2 \mathrm{~b}$ and e) were essentially identical, and the cell population was dense. On the other hand, the exposure of cells to P1 results in a few dead cells (Fig. 2c), while exposure to P2 results in many dead cells (Fig. 2d). The differences in the morphologies of the dead cells must reflect the death processes of cells. For example, the death process due to P1: water leads to cell bursting due to the osmotic pressure difference between the inside and the outside of the cells and thus the dead cells do not retain their initial shapes. In contrast, P2: ethanol causes death by diffusion into the cell interior and immediate denaturation of the proteins, and thus the dead cells essentially retain their initial shapes and density. Similarly, the morphologies of cells tested using the cytotoxic test solvents exhibit some modifications, such as a sparse cell distribution (Fig. 2f) and cell-shape deformation (Fig. 2g). These morphological changes must likewise reflect the death processes of cells, i.e., the cytotoxic factors of the test solvents.

The cytotoxic factors were investigated by calculating the relative energy differences ${ }^{12}$ (REDs) of nontoxic/cytotoxic testsolvent HSPs with respect to the cell-constituent HSP spheres (C1-C7) (see the ESI $\dagger$ ) and the results are summarized in Fig. 3. A RED $<1.0$ means that a test solvent may potentially dissolve (see the ESI $\dagger$ for supporting results by LC-MS) or diffuse into a cell constituent, i.e., the test solvent is likely cytotoxic. All the RED values of the nontoxic test solvents (Fig. 3a) with respect to all the cell constituents were $>1$, which demonstrates that having HSPs outside all the cell-constituent HSP spheres is a necessary condition for solvents to be nontoxic.

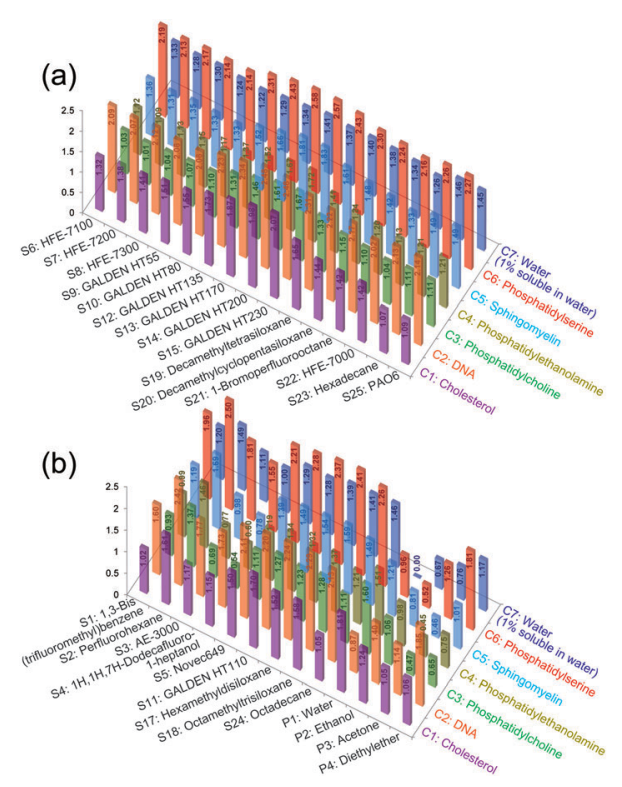

Fig. 3 Relative energy differences (REDs) of (a) nontoxic and (b) cytotoxic solvents with respect to cell constituents.
The RED values of some cytotoxic test solvents (Fig. 3b) with respect to the cell constituents were $\leq 1$. For example, $\mathrm{S} 1$ can dissolve cell-membrane constituents (C3 and C4, RED $<1$ ), and might also dissolve $\mathrm{C} 1$ (RED $\sim 1$ ). Thus, cell death upon contact with S1 likely occurred due to damage to the cell membrane and/or cholesterol (found in the cell membrane). In addition, S3: AE-3000 and S4 can efficiently dissolve cell-membrane constituents (C3-C5, RED « 1) and might diffuse into the cell interior (which has a high concentration of water; C7, RED $\sim 1$ ). Thus, cell death in contact with S3 and S4 probably occurred due to damage/denaturation of the cell membrane and/or internal cell proteins.

However, the RED values of the cytotoxic test solvents S2, S5: Novec649, S11: GALDEN HT110, S17: hexamethyldisiloxane, S18: octamethyltrisiloxane and S24: octadecane with respect to all the cell constituents were $>1$, which suggests that these cytotoxic solvents might represent anomalies in terms of HSP prediction. Since the RED of $\mathrm{S} 24$ with respect to $\mathrm{C} 1$ is $\sim 1$ (S24 might dissolve $\mathrm{S} 1$ ), the result for $\mathrm{S} 24$ should not be attributed to an anomaly. Therefore, only the 5 cytotoxic test solvents S2, S5, S11, S17 and S18 (excluding S24) should be tentatively considered as anomalies and explored to clarify their potential secondary cytotoxic factors. We repeated the direct-contact tests with S2, S5, S11, S17 and S18 to verify the reproducibility of the test results and additionally carried out live/dead cell observation to clarify the secondary cytotoxic factors. Fig. 4a shows the results of the repeated direct-contact tests and the inset photos show the results of live/dead cell observation after the test with S2 and S18 (see the other results in Fig. S6 and S7, ESI $\dagger$ ). Retesting with $\mathrm{S} 2$, S5 and S17 provided essentially the same results as the first test, i.e., moderately cytotoxic, highly cytotoxic and highly cytotoxic, respectively. In contrast, retesting with S11 and S18 showed reduced cytotoxicity compared to the first test.
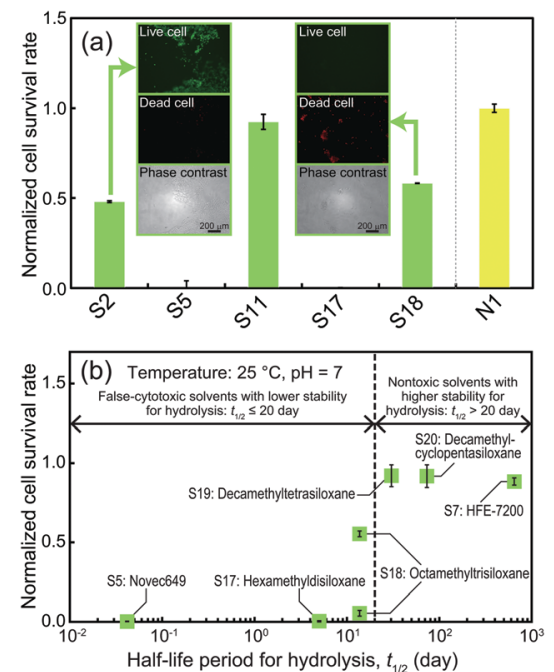

Fig. 4 (a) NCSR after repeating the direct-contact test with solvents identified as anomalies in the first test from HSP prediction. Insets show optical micrographs of cells stained following live/dead cell staining after the test with S2 and S18. (b) Dependence of the normalized cell survival rate with respect to the half-life for the hydrolysis of the solvents in the presence of liquid water. 
As shown in the inset photos for S2 (Fig. 4a) comparing the live/dead cell distribution with the phase contrast results, the phase contrast shows that some cells had detached from the culture well surface and almost all the remaining cells were alive (exhibited green fluorescence). This detachment is attributed to both the high specific gravity and the high hydrophobicity of S2. The hydrophobicity of S2 could result in solvent infiltration into the interface between the cells and the underlying polystyrene of the test well; in addition, the high specific gravity of S2 could result in cells floating due to buoyancy, resulting in the cells detaching from the well surface. This would result in underestimation of the survival rate of cells treated with $\mathrm{S} 2$ and assessed by the WST- 8 assay. Therefore, we conclude that the moderate cytotoxicity of $\mathrm{S} 2$ was a false-positive, i.e., $\mathrm{S} 2$ is inherently a nontoxic solvent and not an anomaly. Cell detachment is also a potential factor affecting the reproducibility of the test with S11 because phase contrast observation (Fig. S7, ESI $\dagger$ ) revealed some detached cells and all the remaining attached cells were alive. Therefore, we also conclude that the moderate cytotoxicity of S11 in the first test was a false-positive, i.e., S11 is inherently a nontoxic solvent and not an anomaly.

On the other hand, the reduced cytotoxicity of S18 in the repeat test should be attributed to an artifact due to non-contact of the test solvent with cells around the periphery of the test wells due to the residual culture medium blocking the cells from the test solvent. The inset photos of cells treated with S18 (Fig. 4a) shows that cells in the center region of the test well were definitely in direct contact with the test solvent and all the cells were dead. Hence, S18 remained categorized as a cytotoxic solvent and as an anomaly.

As confirmed above, S2 and S11 were non-toxic and not anomalies, while S5, S17 and S18 were cytotoxic and anomalies. We suspect the chemical stability of the cytotoxic test solvents to act as a secondary cytotoxic factor: specifically, the degradation products due to hydrolysis of the test solvents caused false cytotoxicity. Hydrolysis data of the half-life $\left(t_{1 / 2}\right)$ of S5, S7, S17, S18, S19: decamethyltetrasiloxane and S20: decamethylcyclopentasiloxane $^{18-23}$ and the NCSR with the solvents were plotted in Fig. 4b. This dependence shows a threshold for $t_{1 / 2}$ at $\sim 20 \mathrm{~h}$ and clearly divides false-cytotoxic from nontoxic solvents. This demonstrates that only less stable solvents with $t_{1 / 2}<20 \mathrm{~h}$ for hydrolysis resulted in false cytotoxicity. S5, S17 and S18 generate alcohols and diols as hydrolytic products, ${ }^{18,20,24}$ and HSPs indicate that these products are generally considered to be cytotoxic. Therefore, the false cytotoxicity must originate from the hydrolytic products of S5, S17 and S18. As discussed above, since all the anomalies were excluded as false-positive (inherently nontoxic) or false-cytotoxic solvents, our hypothesis is highly probable. Furthermore, we propose that the hydrolytic instability of solvents is the secondary cytotoxic (false-cytotoxic) factor.
In conclusion, we have proposed a hypothesis that "nontoxic organic solvents are substances that do not dissolve (or diffuse into) cell constituents", and based on this hypothesis we predicted potentially nontoxic organic solvents in terms of the HSP values of cell-constituents. Nontoxic organic solvents were experimentally identified amongst various low-polar/nonpolar substances. Cytotoxic test solvents predicted as being nontoxic based on HSP consideration, i.e., anomalies, were re-examined. The hydrolytic instability of these solvents and the toxicity of their products were identified as giving rise to secondary cytotoxicity and causing the observed anomalies. The identified nontoxic solvents and the proposed screening method based on HSPs will contribute to a wide range of applications such as 3D cell printing and cell sheet fabrication.

\section{Notes and references}

1 C. P. Carpenter and H. F. Smyth, Am. J. Ophthalmol., 1946, 29, 1363-1372.

2 H. F. Smyth, C. P. Carpenter and C. S. Weil, Am. Ind. Hyg. Assoc. J., 1962, 23, 95-107.

3 W. M. Grant, in Toxicology of the Eye, ed. C. C. Thomas, Springfield, Illinois, 1974.

4 C. J. Kirwin and E. E. Sandmeyer, Ether, Patty's Hygiene and Toxicology, John Wiley \& Sons, New York, 1981.

5 D. M. Pace and A. Elliott, Cancer Res., 1962, 22, 107-112.

6 W. C. Stevens, E. I. Eger, T. A. Joas, T. H. Cromwell, A. White and W. M. Dolan, Can. Anaesth. Soc. J., 1973, 20, 357-368.

7 M. C. Z. Kasuya, X. Wen, K. Hatanaka and K. Akashi, J. Fluorine Chem., 2011, 132, 978-981.

8 L. C. Clark and F. Gollan, Science, 1966, 152, 1755-1756.

9 I. Giaever and C. R. Keese, Proc. Natl. Acad. Sci. U. S. A., 1983, 80, 219-222.

10 H. W. Kang, S. J. Lee, I. K. Ko, C. Kengla, J. J. Yoo and A. Atala, Nat. Biotechnol., 2016, 34, 312-319.

11 N. Yamada, T. Okano, H. Sakai, F. Karikusa and Y. Sakurai, Macromol. Rapid Commun., 1990, 11, 571-576.

12 C. M. Hansen, Hansen solubility parameters: a user's handbook, CRC Press, Boca Raton, 2007.

13 C. M. Hansen, Eur. Polym. J., 2008, 44, 2741-2748.

14 S. Abbott, C. M. Hansen, H. Yamamoto and R. S. Valpey, Hansen solubility parameters in practice, complete with eBook, software and data 4th edition version 4.1, http://www.hansen-solubility.com/, accessed 18, Nov. 2016.

15 J. F. Lechner and M. A. LaVeck, J. Tissue Cult. Methods, 1985, 9, 43-48.

16 J. R. W. Masters, Animal Cell Culture: A Practical Approach, Oxford University Press, USA, 2000.

17 Live/Dead Cell Staining Kits, http://www.promokine.info/products/ cell-analysis/livedead-cell-staining-kits/, accessed 14, Nov. 2016.

18 Octamethyltrisiloxane (L3), http://webnet.oecd.org/hpv/ui/handler. axd?id=83c0a20e-ecb8-4667-8f2d-7a06aaf70e91, accessed 14, Nov. 2016.

19 3M Novec 7200 Engineered Fluid, http://www.acota.co.uk/assets/ data-centre/msds/3m/3mnovec7200.msds.pdf, accessed 14, Nov. 2016.

20 Hexamethyldisiloxane (HMDS), http://webnet.oecd.org/Hpv/UI/handler. axd?id=98264d1f-2476-42fb-ade8-0fc8485bae4c, accessed 14, Nov. 2016.

21 Decamethylcyclopentasiloxane, https://echa.europa.eu/registrationdossier/-/registered-dossier/14807/2/3/?documentUUID=97cf652d-7c2f4815-a153-958b5048d3bb, accessed 17, Nov. 2016.

22 Decamethyltetrasiloxane, https://echa.europa.eu/registration-dossier/-/ registered-dossier/5665/2/3, accessed 17, Nov. 2016.

23 Dodecafluoro-2-methylpentan-3-one, https://echa.europa.eu/registrationdossier/-/registered-dossier/9516/5/2/3, accessed 17, Nov. 2016.

24 3M Novec 649 Engineered Fluid Datasheet, http://multimedia.3m. com/mws/media/5698650/3mtm-novectm-649-engineered-fluid.pdf, accessed 14, Nov. 2016. 